\title{
Typ-Cottini-Minimalvariante der Dermatitis herpetiformis Duhring
}

\section{Type Cottini a Minimal Variant of Dermatitis Herpetiformis Duhring}

Autoren

Institute

\section{S. Lohrisch' ${ }^{1}$ W. Meyer ${ }^{2}$}

1 Praxis für Dermatologie, Kassel

2 Institut für Pathologie Nordhessen, Kassel

\section{Bibliografie}

DOI http://dx.doi.org/ 10.1055/s-0029-1215118

Online-Publikation: 20. 10. 2009

Akt Dermatol 2010; 36:

189-191 ๑) Georg Thieme

Verlag KG Stuttgart · New York ISSN 0340-2541

\section{Korrespondenzadresse}

\section{Dr. Steffi Lohrisch}

Praxis für Dermatologie

Wilhelmshöher Allee 273

34131 Kassel

s.lohrisch@gmx.de

\section{Zusammenfassung \\ $\nabla$}

Es wird ein 66-jähriger Patient mit Blasen an beiden Ellenbogen (Typ Cottini) als Minimalvariante einer Dermatitis herpetiformis Duhring vorgestellt. Sie ist mit einer klinisch stummen Zöliakie

\section{Einleitung}

1884 beschrieb Duhring das nach ihm benannte Krankheitsbild. Aber erst 1955 publizierte Cottini eine Variante dieser blasenbildenden Erkrankung. Sie tritt ausschließlich an Ellenbogen und Knien auf [1]. Es ist die Frage, ob die Minimalvariante ebenfalls mit einer Zöliakie verbunden ist und welche lymphozytäre Zusammensetzung vorliegt.

\section{Anamnese}

Ein 66-jähriger Patient stellte sich mit juckenden Hautveränderungen an beiden Ellenbogen vor, die seit ca. 2 Wochen bestanden.

\section{Dermatologischer Befund}

Am rechten und linken Ellenbogen zeigten sich symmetrisch verteilt mehrere gruppierte, mit seröser, teils hämorrhagischer Flüssigkeit prall gefüllte Vesikel auf erythematösem Grund, wobei es z.T. zu Erosionen gekommen war ( $\bullet$ Abb. 1). Die Knie waren hauterscheinungsfrei.

\section{Histologie}

Subepidermale Blase gefüllt mit eosinophilen und neutrophilen Granulozyten. In den dermalen Papillarkörpern sind eosinophile Mikroabszesse (৫ Abb. 2).
(Zottenatrophie mit Plasmazellen- und Lymphozyten (CD8 > als CD4)-Infiltration (Marsh Typ IIIa) verbunden. Die HLA-Typisierung wies die Typen DR3 und DQ2 nach. Kaliumjodid führte zur Provokation, die glutenfreie Kost in Kombination mit DADPS zur Erscheinungsfreiheit.

\section{Immunhistochemie}

Granuläre Ablagerungen von IgA und IgG zeigen sich in den dermalen Papillarkörpern sowie geringe Mengen von Komplement C3 ( $\bullet$ Abb. 3).

\section{Labor}

Nachweis von: Gliadin-IgG-AK: $163 \mathrm{RE} / \mathrm{ml}(<25)$; Gliadin-IgA-AK: $151 \mathrm{RE} / \mathrm{ml}(<25)$; Gewebstransglutaminase IgA-Ak: 83,7 RE/ml (<20).

Im Normbereich lagen: Jod im Serum: 62,0 ug/l (46-70); Anti-Nukleäre-Antikörper: $<1: 80$ Titer $(<1: 80)$; Centromeren-AK: $<1: 80$ Titer $(<1: 80)$; ds-DNS-AK: 19,0 IE/ml (<100); Immunglobulin-A i.S.: $172 \mathrm{mg} / \mathrm{dl} \quad(40-350) ; \quad$ Endomysium-AK: $<1: 10$ Titer $(1: 10)$; Gewebstransglutaminase IgG-Ak: 0,06 Ratio $(<1,0)$; kein Nachweis von zirkulierenden Antikörpern gegen bullöses Pemphigoid und linearer IgA-Dermatose.

HLA-Typisierung ergab den Nachweis von DR3, DQ2.

\section{Duodenoskopie}

Makroskopisch unauffälliger Befund.

\section{Histologie und Immunhistochemie des tiefen Duodenums ( 0 Abb. 4-6) \\ $\nabla$}

Mikroskopisch fällt neben einer partiellen Zottenatrophie und Kryptenhyperplasie ein plasmazellreiches und lymphozytäres Entzündungsinfiltrat in der Lamina propria auf. Intraepitheliale CD3+-T-Lymphozyten innerhalb des Kryptenepi- 


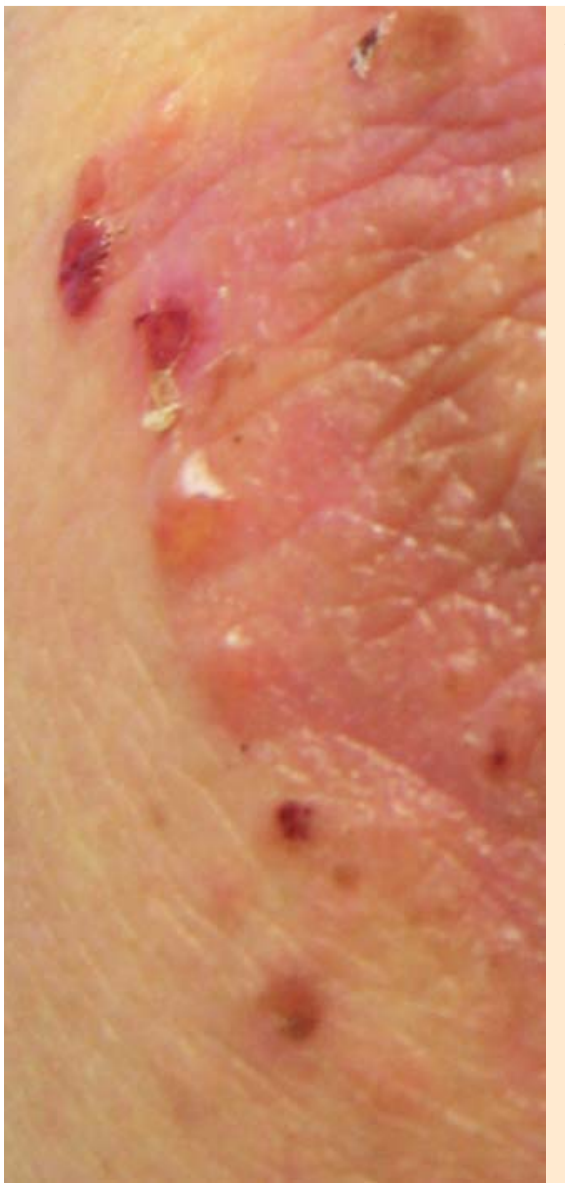

Abb. 1 Rechter Ellenbogen mit prallen serösen, teils hämorrhagischen, teils erodierten Blasen auf erythematösem Grund.

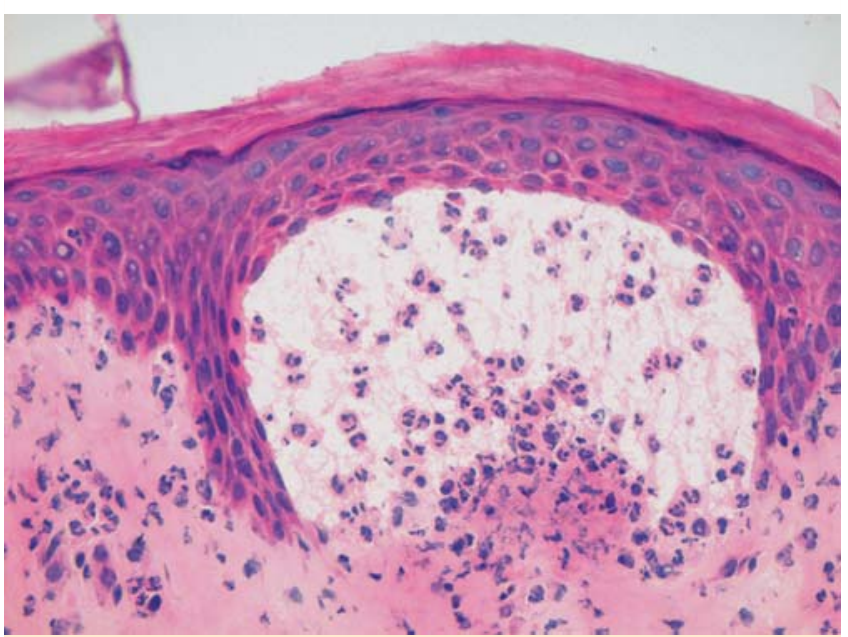

Abb. 2 Blase mit Blasenrand: Histologie: neutrophile und eosinophile Mikroabszesse in den Papillenspitzen mit beginnender subepidermaler Blasenbildung.

thels sind mit ca. $80 \mathrm{IEL} / 100$ Enterozyten (Grenzwert $40 \mathrm{IEL} / 100$ Enterozyten) vermehrt ( $\bullet$ Abb. 4).

Bei der Typisierung der T-Lymphozyten ist ein geringer Anteil von $\mathrm{CD} 4^{+}$-T-Helferzellen $(\bullet$ Abb. $\mathbf{5})$ und ein wesentlich größerer von $\mathrm{CD}^{+}$-T-Suppressorzellen $(\boldsymbol{\bullet} \mathbf{A b b} . \mathbf{6})$ nachweisbar.

Die Plasmazellen exprimieren IgA bzw. IgG.

Die Histologie entspricht einem Typ IIla der Marsh-Klassifikation.

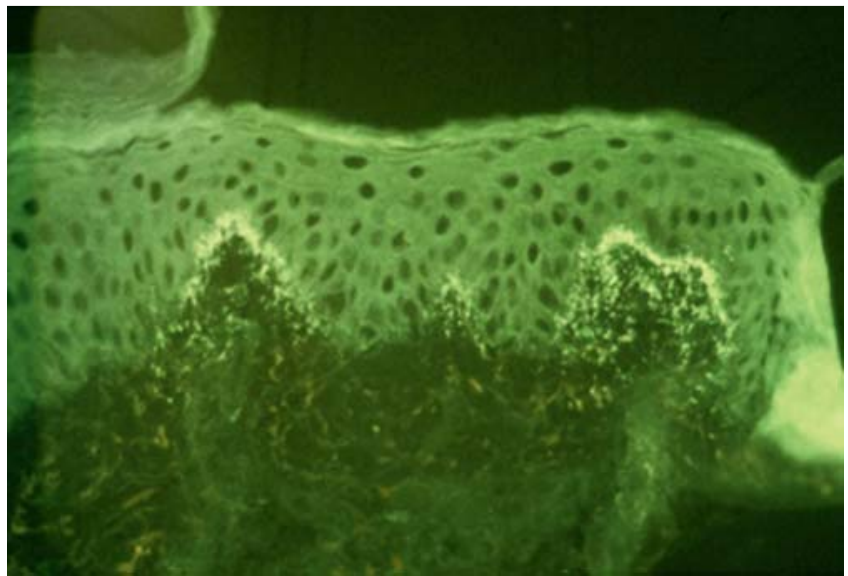

Abb. 3 Blasenrand: direkte Immunfluoreszenz: granuläre Ablagerung von $\lg A$ und $\lg G$ in den dermalen Papillenspitzen sowie geringe Mengen von Komplement C3.

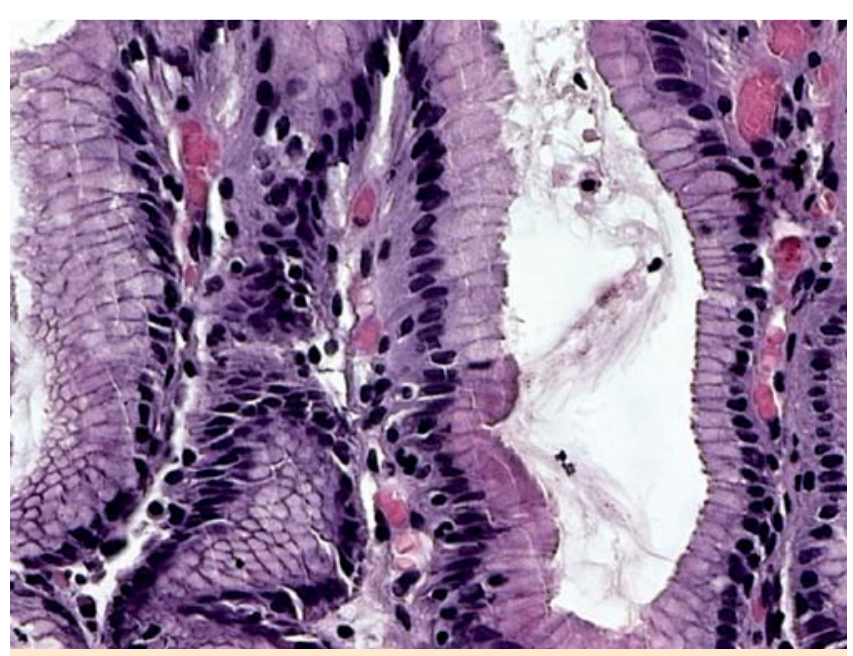

Abb. 4 Histologie des tiefen Duodenums: intraepithelial gelegene Lymphozyten.

\section{Therapie und Verlauf}

\section{$\nabla$}

Wegen einer Schilddrüsenteilresektion erhielt der Patient Thyronajod, welches Kaliumjodid und L-Thyroxin enthält. Nach Umsetzung auf L-Thyroxin und damit Weglassen des freien Kaliumjodids besserte sich das Krankheitsbild deutlich, aber es traten immer noch vereinzelt kleinere Bläschen auf. Erst die Kombination von Dapson und glutenfreier Kost brachte die Erscheinungs- und Rezidivfreiheit.

\section{Diskussion}

$\nabla$

In der Literatur finden sich nur zwei Publikationen des Typs Cottini [2,3]. Dieser Typ muss als Minimalvariante der Dermatitis herpetiformis Duhring angesehen werden. Er tritt nach der Literatur an Ellenbogen und Knien auf [1]. Im vorgestellten Fall waren nur an den Ellenbogen Blasenbildungen vorhanden. Auch der Typ Cottini, wie im geschilderten Fall, ist mit einer glutensensitiven Enteropathie und mit der HLA-DQ2 (DQw2)-Assoziation verbunden. 


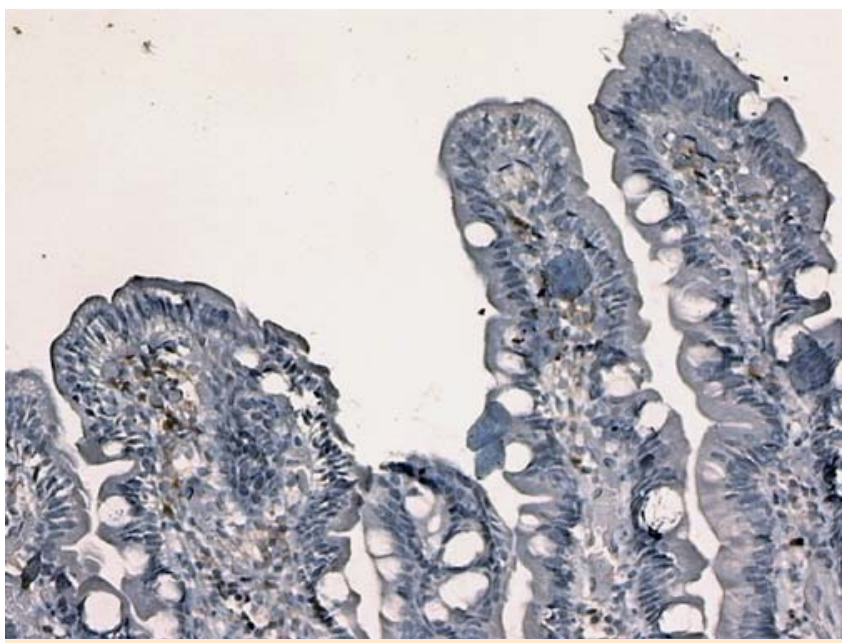

Abb. 5 Immunhistochemie des tiefen Duodenums: CD4+-T-Lymphozyten (T-Helferzellen).

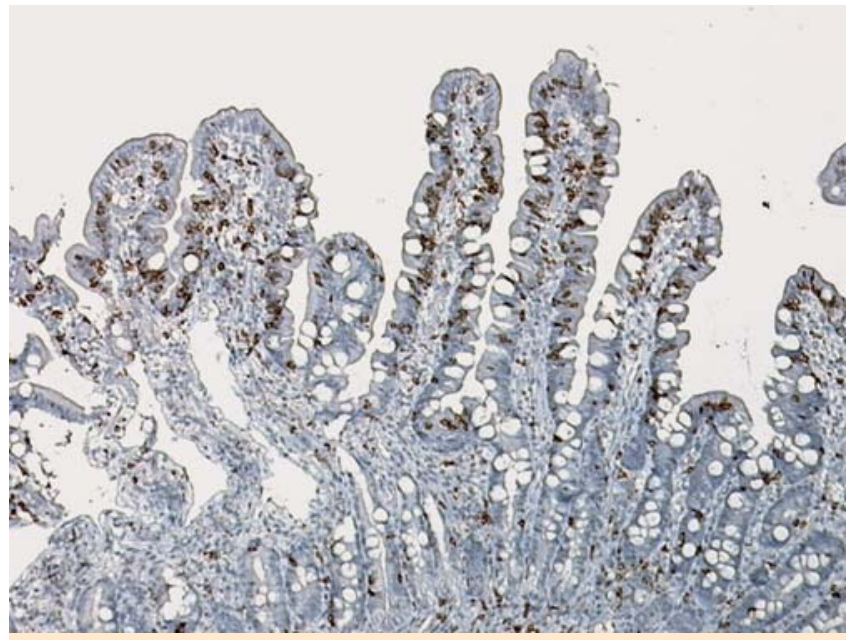

Abb. 6 Immunhistochemie des tiefen Duodenums: CD8+-T-Lymphozyten (T-Supressorzellen).

Die pathogenetischen Abläufe im Duodenum-Jejunum, die zur Zottenatrophie führen, und damit in eine Zöliakie einmünden, sind schon recht gut bekannt. Der HLA-DQ2-Lokus soll zu einer besseren Bindung des $\alpha$-Gliadins, einer Fraktion des Glutens, an die antigenpräsentierenden Zellen führen. Die Passgenauigkeit wird durch die Abspaltung der Amidgruppe von der Glutaminsäure mittels der Gewebstransglutaminase erhöht [4]. Damit wird das Gliadin zum Antigen und die Zöliakie einerseits zur Nahrungsmittelallergie (Bildung von IgA- und IgG-Komplexen) und andererseits durch die Bildung der Gewebstransglutaminase-Antikörper zur Autoimmunerkrankung. Die Zerstörung der Enterozyten und die damit verbundene Einleitung der Zottenatrophie erfolgt sowohl durch die T-Supressorzellen (CD8) (s. - Abb. 6) als auch durch Zytokine [4]. Es ist anzunehmen, dass die vor Ort gebildeten IgG-Immunkomplexe über eine Aktivierung der Komplementkaskade ebenfalls zur Zerstörung beitragen.

Die IgA- wie auch die IgG-Komplexe gelangen vom Darm in den Blutkreislauf. Aber nur in einem Teil der Fälle werden, wie auch im vorgestellten Cottini-Fall, diese Komplexe (hier bevorzugt die IgA-Komplexe) im Stratum papillare der Haut abgelagert (s. - Abb. 3). Anschließend setzen die bekannten Nachfolgereaktionen der Aggregation von Granulozyten, besonders der Eosinophilen, die Aktivierung des alternativen Komplementweges und die Enzymfreisetzung, die dann die subepidermale Blasenbildung verursacht, ein. Offen bleibt die Frage, was die Ablagerung der Antigen-Antikörper-Komplexe verursacht und warum dies nur in der Haut erfolgt.

Wie bei der Dermatitis herpetiformis Duhring so auch beim vorgestellten Cottini-Fall führte die Jodidgabe, obwohl Jod im Serum nicht in erhöhter Konzentration vorlag, zur Exazerbation der Erkrankung.

Es muss daher auch beim Cottini-Typ der Dermatitis herpetiformis neben der reduzierten Jod-Kost eine Allergeneliminationskost (glutenfrei) erfolgen, um somit eine kausale Therapie einzuleiten und um das Risiko der Entwicklung eines intestinalen Lymphoms auszuschalten $[1,5]$. Als therapeutisch aber unverzichtbar darf die Gabe von DADPS (Dapson $50 \mathrm{mg} / \mathrm{d}$ ) angesehen werden.

\section{Abstract}

\section{Type Cottini a Minimal Variant of Dermatitis Herpetiformis Duhring}

We report on a 66 year old patient with blisters on both elbows (type Cottini) as minimal variant of Dermatitis Herpetiformis Duhring. It is associated with a clinically silent celiac disease (villous atrophy with the infiltration of plasma cells and lymphocytes (CD8+> CD4+) (marsh type IIa). The HLA-typing detected the types DR3 and DQ2. Potassium iodide caused provocation. The skin lesions responded well to the combination of glutenfree food and DADPS.

\section{Literatur}

1 Zillikens D. Dermatitis herpetiformis. In: Braun-Falco O, Plewig G, Wolff HH, Burgdorf WHC, Landthaler M (Hrsg). Dermatologie und Venerologie. Heidelberg: Springer, 2005

2 Cottini GB. Dermatite herpetiforme de Duhring symetrique et localise aux genome et aux condes. Ann Derm Syph 1955; 82: 285-286

3 Luderschmidt C, Neubert U. Lokalisierte Dermatitis herpetiformis Duhring Typ Cottini. Hautarzt 1978; 29: 343-344

4 Noffsinger A, Fenoglio-Preiser C, Maru D, Gilinsky N. Celiac disease. In: Noffsinger A, Fenoglio-Preiser C, Maru D, Gilinsky N (Hrsg). Gastrointestinal Diseases. Volume 5. Atlas of nontumor pathology. Washington DC: American Registry of Pathology, 2007: 589-599

5 Ormann W, Hirschmann WD, Alexandrakis E, Bauerle H. Sprue-assoziiertes T-Zell-Lymphom. Dtsch med Wochenschr 1989; 114: 13241327 